\title{
Propriedades mecânicas de painéis produzidos com lascas de madeira em três diferentes comprimentos
}

\author{
Mechanical properties of wood panels produced \\ with wood strands with three different lengths
}

\author{
Emanoele Maria Santos Chiromito ${ }^{1}$, Cristiane Inácio de Campos², \\ Bruno Santos Ferreira ${ }^{3}$, André Luis Christoforo ${ }^{4}$ e Francisco Antonio Rocco Lahr ${ }^{5}$
}

\begin{abstract}
Resumo
O desenvolvimento da tecnologia na produção de painéis e o aumento da demanda por estes produtos, aliado ao encarecimento da madeira maciça de grandes dimensões, contribuíram por motivar o desenvolvimento da indústria de produtos engenheirados de madeira. A partir desta tendência do mercado madeireiro é que se definiu o objetivo do presente trabalho, que consistiu em avaliar as propriedades mecânicas de painéis de partículas orientadas OSB (Oriented Strand Board), OSL (Oriented Strand Lumber) e LSL (Laminated Strand Lumber), produzidos com madeira de Pinus taeda e resina poliuretana à base de óleo de mamona, avaliando-se a influência do comprimento das lascas (inerentes a cada tipo de painel) nas propriedades de rigidez e de resistência. Os ensaios de flexão foram realizados segundo as normas EN 310-2000 e ASTM D 198-09, para a determinação do módulo de elasticidade (MOE) e do módulo de ruptura (MOR) dos três tipos de painéis avaliados. Os resultados do MOE e do MOR, para os três tipos de painéis, atenderam aos requisitos mínimos da norma EN 310-200, e o aumento do comprimento das lascas influenciou positivamente, de forma significativa, nestas propriedades, apresentando os painéis LSL os melhores resultados, seguidos dos painéis OSL e OSB, respectivamente.
\end{abstract}

Palavras-chave: Módulo de elasticidade; módulo de ruptura; Pinus taeda; painéis estruturais.

\begin{abstract}
The development of technology in panel production and the increased demand for these products, coupled with the increased cost of solid wood with large dimensions, led to the development of engineered wood products industry. Because of this, the aim of this research was to evaluate mechanical properties in static bending of Oriented Strand Board (OSB), Oriented Strand Lumber (OSL) and Laminated Strand Lumber (LSL), produced with Pinus taeda wood and castor oil based polyurethane resin. The influence of the strand lengths (inherent in each type of panel) in its properties of strength and stiffness was evaluated. Bending test was carried out according to EN 310-2000 and ASTM D 198-09 standards for determining the modulus of elasticity and modulus of rupture of the three types of panels. The results of strength (MOR) and stiffness (MOE) in bending for the three types of panels met the minimum requirements of EN 310-2000 standard, and the increase in the length of the strands influenced positively and significantly these properties. LSL panels presented the best results, followed by the OSL and OSB panels, respectively.
\end{abstract}

Keywords: Modulus of elasticity; modulus of rupture; Pinus taeda; structural panels

\footnotetext{
${ }^{1}$ Mestranda em Ciências e Engenharia de Materiais. USP - Universidade de São Paulo / EESC - Escola de Engenharia de São Carlos. Avenida Trabalhador são-carlense, 400 - 13566-590 - São Carlos, SP, Brasil. E-mail: emanoelechiromito@usp.br

${ }^{2}$ Professor assistente. UNESP - Universidade Estadual Paulista Júlio de Mesquita Filho / Unidade Diferenciada de Itapeva. Rua Geraldo Alckimin, n. 519 - V. Nossa Senhora de Fátima - 18409-010 - Itapeva, SP, Brasil. E-mail: cristiane@itapeva.unesp.br

${ }^{3}$ Doutorando do Programa de Pós-Graduação em Engenharia Mecânica. UNESP - Universidade Estadual Paulista "Julio de Mesquita Filho / FEG - Faculdade de Engenharia de Guaratinguetá. Av. Ariberto Pereira da Cunha, 333 - 12.516-10 - Guaratinguetá, SP, Brasil. Email: brunosferreira@gmail.com

${ }^{4}$ Professor Adjunto do Departamento de Engenharia Civil. UFSCar - Universidade Federal de São Carlos. Rodovia Washington Luís, km 235 - 36307352 - São Carlos, SP, Brasil. E-mail: christoforoal@yahoo.com.br

${ }_{5}^{5}$ Professor Titular do Departamento de Engenharia de Estruturas. USP - Universidade de São Paulo / EESC - Escola de Engenharia de São Carlos. Avenida Trabalhador são-carlense, 400 - 13566-590 - São Carlos, SP, Brasil. E-mail: frocco@sc.usp.br
} 


\section{INTRODUÇÃO}

Os painéis à base de madeira apresentam crescente aplicação em diversos segmentos, podendo-se destacar a indústria moveleira e a construção civil. Acompanhando esse crescimento observa-se a necessidade cada vez maior de desenvolver um material mais sustentável e racional que atenda as necessidades do segmento industrial a que se destina, garantindo a mesma durabilidade apresentada pelos materiais concorrentes. Os produtos à base de madeira tem apresentado crescimento tanto na produção quanto aplicações em diversos segmentos. Isto se deve ao surgimento de novas tecnologias que permitem uso mais racional da madeira, especialmente espécies de rápido crescimento e dimensões reduzidas (NASCIMENTO et al., 2015).

Em paralelo a isso, observa-se que a construção civil é um segmento industrial que ainda necessita de maior industrialização, de modo a racionalizar e agilizar o processo produtivo, como acontece em outros segmentos industriais. Ressalta-se que sistemas construtivos que empregam a madeira e seus produtos engenheirados são bastante comuns na América do Norte, em aproximadamente $90 \%$ das habitações unifamiliares utilizam a madeira como material de construção (APA, 2011). Isso pode ser justificado especialmente pela rapidez e limpeza durante a execução, elevado desempenho termo-acústico além de custo competitivo apresentado por esse material.

O grande crescimento das indústrias de painéis no Brasil é notável no mercado mundial, tornando-se um dos segmentos mais importantes no âmbito florestal, e isso só se tornou possível devido as excelentes condições para o plantio de florestas de rápido crescimento e grandes áreas para esse fim. Além disso, destaca-se o avanço tecnológico na fabricação dos mais diversos tipos de painéis para os mais variados usos. O fato de a madeira ser uma matéria-prima renovável e necessitar de baixo consumo de energia para ser processada, esta representa vantagens em relação a outros produtos de mesma aplicação (MENDES, 2001). Dentro desse contexto, destaca-se a grande viabilidade de uso de alguns painéis, especialmente na construção civil, podendo-se destacar o Oriented Strand Board - OSB (SOUZA et al., 2014; NASCIMENTO et al., 2015), o Oriented Strand Lumber - OSL e o Laminated Strand Lumber - LSL.

Segundo Maloney (1993), o OSB é um painel estrutural produzido a partir de lascas (strands) de madeira, sendo a camada interna disposta aleatoriamente, ou perpendicularmente às camadas externas, ou ainda, como um painel constituído por camadas de lascas ou tiras alinhadas em número ímpar. O OSB tem suas partículas com aproximadamente $25 \mathrm{~mm}$ de largura, de 80 a120 $\mathrm{mm}$ de comprimento e com espessura entre 0,5 e 0,75mm (IWAKIRI, 2005). O OSL é um painel estrutural produzido com partículas com $150 \mathrm{~mm}$ de comprimento, que são unidas a partir de um adesivo estrutural e, posteriormente, orientadas paralelamente (BAO, 2002). O LSL, por sua vez, é um painel estrutural produzido com lascas de madeira, com espessura variando de 0,6 a 1,3 mm, comprimento de $300 \mathrm{~mm}$ e larguras variáveis, orientadas paralelamente.

As placas de OSB têm a função de contraventar a estrutura de paredes, coberturas, mezaninos, lajes secas, plataforma de pisos e forros, sendo também utilizadas na fabricação de embalagens e móveis. Uma grande vantagem das chapas de OSB é que as mesmas são leves, simples e rápidas de serem montadas. Do mesmo modo que o OSB, os painéis OSL e LSL também são amplamente utilizados na construção civil como elementos estruturais de elevado desempenho mecânico, em especial, como elementos de vigas e estruturas de cobertura (APA, 2011).

Nos painéis de lascas, uma relação importante que deve ser considerada na fabricação das chapas é referente a razão de esbeltez, que consiste na relação entre o comprimento e a espessura da partícula (IWAKIRI, 2005). Painéis com a mesma espessura e variação de comprimento indicam que os melhores resultados são conseguidos em painéis produzidos com partículas mais longas (IRLE, 2012).

Moslemi (1974) estudou a relação entre a razão de esbeltez e as propriedades físicas e mecânicas dos painéis produzidos, na qual se observou que a menor razão de esbeltez resultou em menores valores do módulo de resistência na flexão estática, maior inchamento em espessura e maior absorção de água, tornando o material menos resistente e mais instável. No entanto, segundo o mesmo autor, para valores de razão de esbeltez superiores a 300 os resultados tenderam a ser desfavoráveis.

Segundo Chirasatitsin et al. (2005), o aumento no comprimento da partícula apresentou melhores resultados para os painéis submetidos aos testes de flexão estática. A geometria das partículas é um dos fatores básicos a afetar as propriedades mais importantes do painel e seu processo de pro- 
dução (BRUMBAUGH, 1960; GATCHELL et al., 1966; VITAL; WILSON, 1980), juntamente com o tipo de madeira, o tipo e a quantidade de adesivo, os aditivos, a estrutura do painel formado pela orientação das partículas, a disposição das camadas e as condições de prensagem do colchão. Tais afirmações são confirmadas quando se compara os resultados dos painéis OSL e dos painéis OSB, em que um aumento no comprimento das lascas utilizadas proporcionam consideráveis melhorias em algumas propriedades mecânicas nos painéis produzidos. Assim, Irle (2012) afirma que as partículas mais compridas são as melhores para a maior parte das propriedades dos painéis.

Este trabalho teve como objetivo avaliar o módulo de elasticidade e o módulo de ruptura na flexão estática de painéis OSB, OSL e LSL fabricados com lascas de Pinus taeda e resina poliuretana à base de óleo de mamona, possibilitando investigar a influência do comprimento das lascas (inerentes a cada tipo de painel) nas propriedades dos painéis estudados.

\section{MATERIAL E MÉTODOS}

Os materiais utilizados foram lascas de Pinus taeda, obtidas por meio de peças residuais de madeira provenientes de indústria de laminação localizada na cidade de Itararé, estado de São Paulo. O adesivo utilizado para a fabricação dos painéis foi a resina poliuretana à base de óleo de mamona, bi-componente, teor de sólidos de $100 \%$, sendo o componente A um poliol derivado do óleo vegetal, com densidade de $1,2 \mathrm{~g} / \mathrm{cm}^{3}$, e o componente $\mathrm{B}$ o isocianato polifuncional, com densidade de $1,24 \mathrm{~g} / \mathrm{cm}^{3}$, fornecido pela Plural Indústria Química.

As lascas foram secas em estufa até atingirem teor de umidade entre 3 e $6 \%$. Os painéis foram produzidos nas dimensões de $500 \times 500 \times 20 \mathrm{~mm}$ e com massa específica nominal de $0,72 \mathrm{~g} / \mathrm{cm}^{3}$. Após a aplicação do adesivo, o colchão foi formado em camadas orientadas, tendo para o OSB três camadas, a intermediária defasada de $90^{\circ}$ em relação às camadas externas, na proporção de materiais de 30: 40: 30 (face: miolo: face), com as lascas orientadas por um sistema de telas (Figura 1). Para os painéis OSL e LSL, o sistema de telas orientadas também foi utilizado, entretanto, com todas as lascas orientadas em um único sentido, mantendo a característica do painel. Ressalta-se que a largura e a espessura das lascas de madeira foram mantidas as mesmas, ou seja, $20 \mathrm{~mm}$ de largura para os três tipos de painéis, assim como a espessura das lascas, com 2,0 mm. O que variou para cada tipo de painel foi o comprimento das lascas (inerentes a cada tipo de painel), sendo para os painéis OSB de $120 \mathrm{~mm}$, para os painéis OSL de $150 \mathrm{~mm}$ e para os painéis LSL de $300 \mathrm{~mm}$.

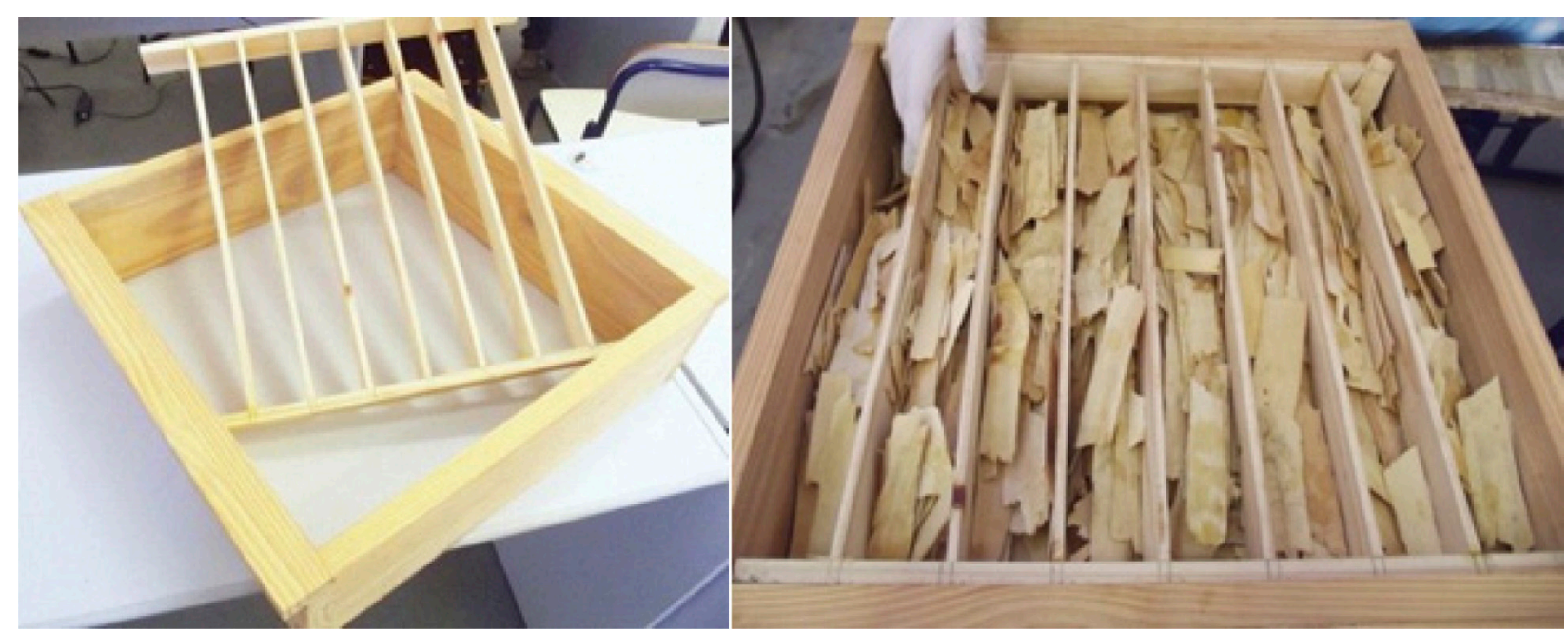

Figura 1. Caixa formadora para a disposição das lascas e sistema de tela para facilitar a orientação do material. Figure 1. Box and grid system for the arrangement and orientation of the strands.

Em seguida foi realizada a prensagem a quente dos painéis por um período de 10 minutos, com alivio de vapores aos 5 minutos, com temperatura de $150^{\circ} \mathrm{C}$, e pressão específica constante de 40 $\mathrm{kgf} / \mathrm{cm}^{2}$, sendo o equipamento utilizado da marca Hidral-Mac, modelo HNP 80T.

Foram produzidos 6 painéis OSB, 6 painéis OSL e 6 painéis LSL, sendo retiradas 6 corpos de prova de prova cada painel produzido, perfazendo 36 corpos de prova por tipo de painel (OSB, OSL e LSL). A Figura 2 apresenta os três painéis produzidos. 

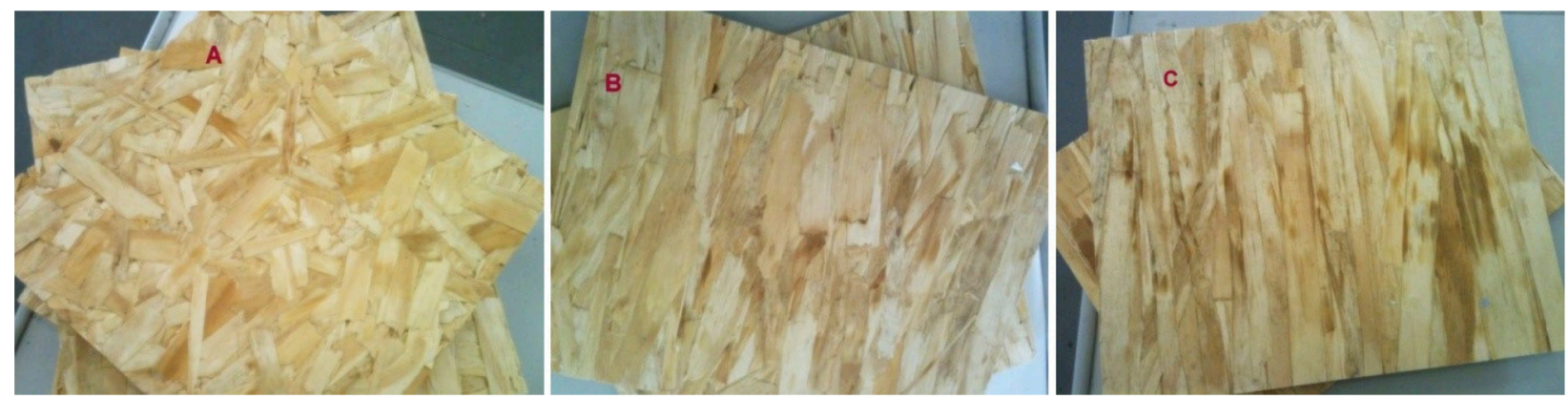

Figura 2. Painéis produzidos: (A) OSB; (B) OSL; (C) LSL.

Figure 2. Produced Panels: (A) OSB; (B) OSL; (C) LSL.

Os painéis foram acondicionados por 72 horas em sala climatizada e, posteriormente, retirados os corpos de prova nas dimensões de $350 \times 50 \times 15 \mathrm{~mm}$, na direção paralela às fibras da camada externa do painel, de acordo com a EN 310-2000. O teste de caracterização mecânica para determinação do módulo de elasticidade (MOE) e módulo de ruptura (MOR) na flexão estática foi realizado de acordo com a norma ASTM D 198-09, com um vão de 300 mm, aplicação de força central e dois apoios laterais (Figura 3).

Foram determinados também o teor de umidade e a densidade dos painéis de acordo com a norma EN 310-2000. Produziu-se 12 corpos de prova para cada teste, com dimensões de 50×50×15 mm.

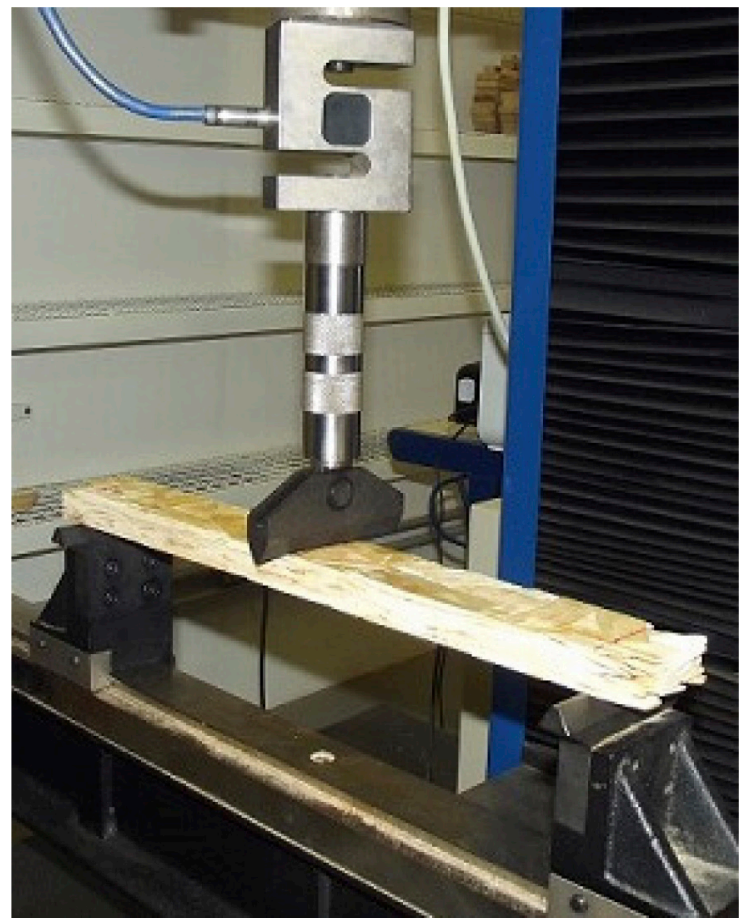

Figura 3. Teste de flexão estática.

Figure 3. Static bending test.

Para investigar a influência do tipo de painéis (ou do comprimento das lascas) nas propriedades de resistência e de rigidez na flexão foi-se utilizada a análise de variância, considerada ao nível de $5 \%$ de significância, sendo utilizados os testes de Anderson-Darling e de Bartlett para verificação da normalidade na distribuição dos resíduos por resposta e a homogeneidade das variâncias dos resíduos entre os tratamentos, respectivamente. Em caso de diferenças estatisticamente significativas entre as propriedades de rigidez ou de resistência dos três tipos de painéis, na sequencia utilizou-se o teste de comparações múltiplas de Tukey, com o objetivo do agrupamento dos seus níveis.

\section{RESULTADOS E DISCUSSÃO}

Os valores médios e os coeficientes de variação dos módulos de elasticidade e de resistência à flexão, assim como os valores das razões de esbeltez para os três tipos de painéis são apresentados na Tabela 1. 
Tabela 1. Médias e coeficientes de variação (\%) do MOE e do MOR, valores da razão de esbeltez (RE), teor de umidade e densidade para os três tipos de painéis fabricados.

Table 1. Mean values and variation coefficient (\%) of MOE and MOR, slenderness ratio (RE), moisture content and density for the three types of panels produced.

\begin{tabular}{lccccc}
\hline Tipo de Painel & TU (\%) & Densidade $\left(\mathbf{g} / \mathbf{c m}^{\mathbf{3}}\right)$ & MOE $(\mathbf{M P a})$ & MOR (MPa) & RE \\
\hline LSL & $10,17 \mathrm{~A}^{*}$ & $0,64 \mathrm{~A}^{*}$ & $12062(19 \%) \mathrm{A}$ & $57,46(23 \%) \mathrm{A}$ & 150 \\
OSL & $10,25 \mathrm{~A}$ & $0,62 \mathrm{~A}$ & $7012(15 \%) \mathrm{B}$ & $46,23(18 \%) \mathrm{B}$ & 75 \\
OSB & $11,03 \mathrm{~A}$ & $0,71 \mathrm{~A}$ & $5775(17 \%) \mathrm{C}$ & $29,06(20 \%) \mathrm{C}$ & 60 \\
\hline
\end{tabular}

*Letras iguais implicam em tratamentos com médias estatisticamente equivalentes, e sendo $\mathrm{A}$ a maior média, $\mathrm{B}$ a segunda maior média e etc.

Cabe destacar que a norma européia EN 310-2000 indica valores mínimos (requisitos) para painéis OSB/3 - Painéis para fins estruturais usados em ambiente úmido, com $4800 \mathrm{MPa}$ para o MOE e $26 \mathrm{MPa}$ para o MOR, e em comparação com os resultados apresentados na Tabela 1, o painel menos resistente, que foi OSB, foi capaz de atender aos requisitos normativos.

Com relação à razão de esbeltez (RE) dos painéis (Tabela 1), ressalta-se que os resultados obtidos estão em conformidade com os discutidos por Moslemi (1974), que afirma que os menores valores das razões de esbeltez estão relacionados aos menores valores do módulo de ruptura na flexão estática, até valores de RE inferiores a 300, como obtido neste estudo.

Os resultados dos testes de normalidade e de homogeneidade entre variâncias apresentaram, para ambas as propriedades investigadas, P-valor superior a 5\%, validando o modelo de ANOVA.

Os resultados da ANOVA evidenciaram a diferença significativa $(\mathrm{P}$-valor $<0,05)$ entre as médias dos valores dos módulos de elasticidade e de ruptura na flexão estática entre os tipos de painéis, revelando ser influente o comprimento das lascas (ou tipo de painel) nestas propriedades. Do teste de Tukey (teste de contraste entre médias), tanto no MOE quanto no MOR os painéis LSL apresentaram os maiores valores médios, seguidos sucessivamente dos valores médios do módulo de elasticidade dos painéis OSL e OSB, respectivamente, evidenciando a superioridade dos painéis LSL frente ao OSL e OSB, o que indica que os maiores comprimentos das lascas forneceram os melhores resultados para as propriedades mecânicas investigadas, resultado esse em conformidade com os obtidos das pesquisas de Chirasatitsin et al. (2005), que discutem que o aumento no comprimento da partícula tem resultado no aumento dos valores das propriedades de flexão estática do painel, e de Irle (2012), que conclui que o maior comprimento das partículas, que podem ser entendidas por lascas, proporcionaram melhorias nas propriedades mecânicas dos painéis.

\section{CONCLUSÕES}

Os resultados obtidos para os três tipos de painéis possibilitam concluir que:

- os painéis com os maiores comprimentos das lascas (LSL) apresentaram os maiores valores para ambas as propriedades investigadas, seguidos, com valores decrescentes, pelos painéis OSL e OSB, respectivamente, resultados esses que estão em conformidade com os obtidos em literatura correlata;

- todos os painéis atenderam aos requisitos estipulados pela norma européia EN 310-2000 para a rigidez e resistência na flexão estática, sendo classificados como OSB/3 - Painéis para fins estruturais usados em ambiente úmido, o que evidencia, também, o excelente desempenho da resina poliuretana bicomponente a base de mamona;

- os menores valores da razão de esbeltez (RE), assim como encontrado na literatura, apresentaram os menores valores da resistência na flexão estática, para RE inferior a 300.

Pela superioridade dos resultados das propriedades na flexão estática obtidas dos painéis LSL e OSL, em relação aos painéis OSB, estes se apresentam como soluções alternativas e de grande potencial para aplicação na construção civil no país, visto que apenas os painéis OSB são fabricados no Brasil para este uso.

\section{AGRADECIMENTOS}

Os autores agradecem a Fundação de Amparo à Pesquisa do Estado de São Paulo (FAPESP). 
Ferreira et al. - Propriedades mecânicas de painéis produzidos com lascas de madeira em três diferentes comprimentos

\section{REFERÊNCIAS BIBLIOGRÁFICAS}

APA - THE ENGINEERED WOOD ASSOCIATION. Engineered Wood Construction Guide Excerpt: Structural Composite Lumber Excerpt from the Engineered Wood Construction Guide. 2011.

BAO, Z. Structural composite lumber. In: APA Engineered wood handbook. New York: Mc Graw-Hill, 2002. chap. 6, p. 6.1-6.49.

BRUMBAUGH, J. Effect of flake dimensions on properties of particleboard. Forest Products Journal, Madison, v. 10, n. 5 , p. $243-246,1960$.

CHIRASATITSIN, S.; PRASERTSAN, S.; WISUTMETHANGOON, W.; KYOKONG, B. >Mechanical properties of rubberwood oriented strand lumber (OSL): The effect of strand length. Songklanakarin Journal of Science and Technology, v. 27, n. 5, p. 1047-1055, 2005.

GATCHELL, C. J.; HEEBINK, B. G.; HEFTY, F. V. Influence of components variables on properties of particleboard for exterior use. Forest Products Journal, Madison, v. 16, n. 4, p. 46-59, 1966.

IRLE, M. A.; BARBU, M. C.; REH, R.; BERGLAND, L.; ROWELL, R. M. Wood Composites. In: ROWELL, R. M. Handbook of wood chemistry and wood composites. Boca Raton: CRC Press, 2012. Cap. 10.

IWAKIRI, S. Painéis de madeira reconstituída. Curitiba: FUPEF, 2005. 247 p.

MALONEY, T. M. Modern particleboard and dry-process fiberboard. 2.ed. San Francisco: Miller Freeman, 1993. $681 \mathrm{p}$.

MENDES, L. M. Pinus spp. na produção de painéis de partículas orientadas (OSB). 2001. 163 p. Tese (Doutorado em Ciências Florestais) - Universidade Federal do Paraná. Curitiba. 2001.

MOSLEMI, A. A. Particleboard. Carbondale: Southern Illinois University Press, 1974. v. 2, 245 p.

NASCIMENTO, M. F.; BERTOLINI, M. S.; CHRISTOFORO, A. L.; PANZERA, T. H.; ROCCO, F. A. L. Painéis OSB fabricados com madeiras da caatinga do nordeste do Brasil. Ambiente Construído, Porto Alegre, v. 15, n. 1, p. 41-48, 2015. Disponível em: < http://www.scielo.br/scielo.php?pid=S1678-86212015000100041\&script=sci

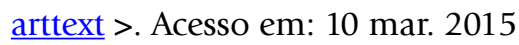

SOUZA, A. M.; VARANDA, L. D.; CHRISTOFORO, A. L.; NASCIMENTO, M. F.; POLETO, S. F. S.; PANZERA, T. H.; ROCCO, F. A. L. Modulus of Elasticity in Static Bending for Oriented Strand Board (OSB). International Journal of Composite Materials, v. 4, p. 56-62, 2014.

VITAL, B. R.; WILSON, J. B. Efeito da forma geométrica dos flocos e partículas, da densidade das chapas e do tipo de adesivo nas propriedades mecânicas das chapas de madeira aglomerada. Revista Árvore, Viçosa, v. 4, n. 2, p. 179-187, 1980.

Recebido em 13/05/2015

Aceito para publicação em 12/08/2015 